\title{
Temporal regularities of changing magnetic field generation modes in the model of the $\alpha \Omega$-dynamo.
}

\author{
Olga Sheremetyeva ${ }^{1, \star}$ and Anna Godomskaya ${ }^{2, \star \star}$ \\ ${ }^{1}$ Institute of Cosmophysical Research and Radio Wave Propagation FEB RAS, Paratunka, Russia \\ ${ }^{2}$ Municipal budgetary institution of supplementary education «Center «Luch»», Yelizovo, Russia
}

\begin{abstract}
In the dynamic model $\alpha \Omega$-dynamo the change in the intensity of the $\alpha$ generator under the action of external forces is considered as a result of synchronization of higher modes of the velocity field and the magnetic field and is regulated by the function $Z(t)$ with an exponential-power kernel $J(t)$. Depending on the choice of the exponent and the scale factor of the kernel determine its temporal characteristics: delay time and waiting time. The question of changing the modes of magnetic field generation depending on the temporal characteristics of the function's kernel is investigating. In the dynamic model $\alpha \Omega$-dynamo the change of the $\alpha$-generator intensity under the action of external forces is considered as a result of synchronization of higher modes of the velocity field and the magnetic field and is regulated by the function $Z(t)$ with an exponential-power kernel $J(t)$. Depending on the choice of the exponent and the scale factor of the kernel $J(t)$ determine its temporal characteristics: delay time and waiting time. The question of changing the modes of magnetic field generation depending on the temporal characteristics of the function's kernel is investigating.
\end{abstract}

\section{Introduction}

The magnetic fields of planets, stars, galaxies undergo changes over time, which are both chaotic and periodic characters. The study of the nature of the occurrence of a magnetic field and the modeling of various modes of its generation, including with a sharp change in the polarity of the field dipole component (reversals), is an intensively developed section of the dynamo theory. Reversals without significant rearrangement of the motion of the conducting medium are of the great interest. The study is carried out both using direct numerical modeling, and on the basis of simplified low-dimensions models, which try to explain the physical cause, signs, and most important properties of this phenomenon [1-3]. In our work, we use the low-mode model $\alpha \Omega$-dynamo [4] to study the modes of magnetic field generation, including those with a change of polarity without convection rearrangement. This model is applied to earth-type objects, which are characterized by a strong differential rotation of the object itself and the turbulent nature of the movement of the conducting medium $[1,2,5]$. In the framework to this model the research is carried out using a dimensionless magnetohydrodynamic system (MHD-system) with a time scale comparable to the time of magnetic field dissipation. The introduction of a variable intensity $\alpha$-generator should ensure the appearance of new modes of magnetic field

${ }^{\star}$ Corresponding author: olga.v.sheremetyeva@gmail.com

${ }^{\star}$ Corresponding author: anna_antonenko@mail.ru 
generation. This article is devoted to the numerical simulation dimensionless MHD-system within the accepted constraints of the $\alpha \Omega$-dynamo model subject to additive correction of the $\alpha$-effect intensity by the integrated function with exponential-power kernel and to the study dynamics of changing generation modes of the magnetic field depending on its temporal characteristics

\section{Formulation of the problem}

We consider the model $\alpha \Omega$-dynamo, in which a spherical shell with the center at the origin, bounding the liquid core of the Earth, rotates around the axis $O z$ with a constant angular velocity $\mathbf{\Omega}$.

The velocity field $\mathbf{v}$ of a viscous incompressible fluid of the outer core and the generated magnetic field are axially symmetric. We assume that the average flow $\overline{\mathbf{v}}$ has the character of differential rotation in the field of external forces with mass density $\mathbf{f}_{\text {out }}[1,2]$.

The inner boundary of the liquid core shell has a radius of $r=r_{1}$, and the outer boundary has a radius of $r=r_{2}\left(r_{1}<r_{2}\right)$, at which the velocity field of the viscous liquid $\mathbf{v}$ is zero (conditions of adhesion). The magnetic permeability of the inner and outer cores is the same, the medium outside the outer core $\left(r>r_{2}\right)$ is non-conducting.

The physical parameters of the shell are assumed to be unchanged, the turbulence in the core is isotropic. The turbulent $\alpha$-effect is antisymmetric relativly to the equator and we use its scalar parameterization as a function $\alpha(r, \theta)=\alpha_{0} a(r) \cos \theta=\alpha_{0} \alpha^{\prime}(r, \theta)$, where the positive coefficient $\alpha_{0}$ determines the intensity of the $\alpha$-effect, $\alpha^{\prime}(r, \theta)$ - dimensionless value, the radial component of $a(r)$ satisfies the condition $\max |a(r)| \sim 1$.

In accordance with the accepted provisions, the dynamo model will be described by MHDequations, including the Navier-Stokes equation, the magnetic field $\mathbf{B}$ induction equation taking into account the turbulent $\alpha$-effect, continuity condition of the velocity field $\mathbf{v}$, the magnetic field solenoidality condition, and boundary conditions. In the Boussinesq approximation, the MHD-system takes the following form

$$
\begin{aligned}
& \frac{\partial \mathbf{v}}{\partial t}+(\mathbf{v} \nabla) \mathbf{v}+\mathbf{f}_{c}=v \Delta \mathbf{v}-\frac{1}{\rho_{0}} \nabla P-\mathbf{f}_{K}+\mathbf{f}_{o u t}+\mathbf{f}_{L}, \\
& \frac{\partial \mathbf{B}}{\partial t}=\nabla \times(\mathbf{v} \times \mathbf{B})+\nabla \times(\alpha(r, \theta) \mathbf{B})+v_{m} \Delta \mathbf{B}, \\
& \nabla \mathbf{v}=0, \\
& \nabla \mathbf{B}=0, \\
& \mathbf{v}\left(\mathbf{r}_{1}\right)=\mathbf{v}\left(\mathbf{r}_{2}\right)=\mathbf{0},
\end{aligned}
$$

where $P$ is pressure, $\rho_{0}=7 \cdot 10^{3} \mathrm{~kg} / \mathrm{m}^{3}$ - density, $v$ - kinematic viscosity (limits of change $10^{-6} \div$ $10^{2} \mathrm{~m}^{2} / \mathrm{sec}$ ), $v_{m}$ - magnetic viscosity (varies within $1 \div 20 \mathrm{~m}^{2} / \mathrm{sec}$ ), $\mathbf{f}_{\text {out }}$ - mass density of the external forces field (source of poloidal velocity), $\mathbf{r}_{1}$ and $\mathbf{r}_{2}$ - radii-vectors of the inner and outer boundaries of the spherical shell of the liquid core, respectively, mass density of the Coriolis force

$$
\mathbf{f}_{K}=2 \mathbf{\Omega} \times \mathbf{v},
$$

the mass density of the Lorentz force

$$
\mathbf{f}_{L}=\frac{1}{\rho_{0} \mu_{0} \mu}(\nabla \times \mathbf{B}) \times \mathbf{B},
$$

the acceleration of the centrifugal force

$$
\mathbf{f}_{c}=\mathbf{\Omega} \times(\boldsymbol{\Omega} \times \mathbf{r}),
$$


$\mu_{0}=4 \pi \cdot 10^{-7} \mathrm{H} / \mathrm{m}$ is magnetic constant, $\mu=1$ - magnetic permeability, the angular velocity $\Omega_{0}$ varies within $10^{-1} \div 101 / \mathrm{sec}$.

The system (1) at constant intensity $\alpha$-effect $\alpha_{0}$ defines the damped and regular modes and unlimited increase of the field (Fig. 2 a). The change of the intensity $\alpha_{0}$ over time under the action of external forces is determined by including to the dynamic system (1) additive correction as a function

$$
Z(t)=\int_{0}^{t} J(t-\tau) Q(\mathbf{B}(\tau), \mathbf{B}(\tau)) d \tau .
$$

The function (5) has the velocity dimension [m/sec], as does the function $\alpha(r, \theta)$, and is used to regulate the degree of turbulent effect influence by delaying and/or inhibition its impact. The nature of such an impact is determined by the dimensionless kernel $J(t-\tau)$.

We introduce the characteristic values of the speed $u_{0}[\mathrm{~m} / \mathrm{sec}]$ and the linear dimension of the area $r_{2}[\mathrm{~m}]$ (radius of the outer core). As a unit of time, we use the dissipation time of the magnetic field in the absence of external influences $L^{2} / v_{m}$ [sec] (the order is $10^{4}$ years). The transformation to new timescale is defined by the ratio $t=\frac{L^{2}}{v_{m}} t^{\prime}$. To reduce the number of variable system parameters, we choose the values of magnetic induction $B_{0}[\mathrm{~T}]$, pressure $P_{0}[\mathrm{PA}]$, the mass density of external forces $f_{0}[\mathrm{~N} / \mathrm{kg}]$ so that the coefficients in the Navier-Stokes equation for the pressure gradient, the mass density of external forces and the Lorentz force were equal to ones. Taking into account that $(\mathbf{v} \nabla) \mathbf{v}=0$, becouse in the model a normal modes of the small amplitude for a viscous rotating liquid is considers [4], the MHD-system (1) in a dimensionless form shall written as follows

$$
\begin{aligned}
& \frac{\partial \mathbf{v}}{\partial t}=P_{m} \Delta \mathbf{v}-\nabla P-E^{-1} P_{m}\left(\mathbf{e}_{z} \times \mathbf{v}\right)+\mathbf{f}_{\text {out }}+(\nabla \times \mathbf{B}) \times \mathbf{B}, \\
& \left.\frac{\partial \mathbf{B}}{\partial t}=R e_{m}[\nabla \times(\mathbf{v} \times \mathbf{B})]+\left(R_{\alpha}-Z(t)\right)\left[\nabla \times \alpha^{\prime}(r, \theta) \mathbf{B}\right)\right]+\Delta \mathbf{B}, \\
& \nabla \mathbf{v}=0, \\
& \nabla \mathbf{B}=0, \\
& \mathbf{v}\left(\frac{\mathbf{r}_{1}}{r_{2}}\right)=\mathbf{v}\left(\mathbf{e}_{2}\right)=\mathbf{0} .
\end{aligned}
$$

\section{The numerical simulation}

We use a low-mode approximation for the numerical implementation of the system (6). Let's limit to the minimum number of modes sufficient to obtain reversals in the model $\alpha \Omega$-dynamo with variable intensity $\alpha$-generator [6-9],

$$
\begin{gathered}
\mathbf{v}=u(t) \mathbf{v}_{\mathbf{0}}(\mathbf{r})=u(t)\left(\alpha_{1} \mathbf{v}_{0,1,0}^{T}+\alpha_{2} \mathbf{v}_{0,2,0}^{P}+\alpha_{3} \mathbf{v}_{0,3,0}^{T}+\alpha_{11} \mathbf{v}_{1,1,0}^{T}+\alpha_{13} \mathbf{v}_{1,3,0}^{T}\right), \\
\mathbf{B}=B_{2}^{T}(t) \mathbf{B}_{0,2,0}^{T}(\mathbf{r})+B_{1}^{P}(t) \mathbf{B}_{0,1,0}^{P}(\mathbf{r})+B_{3}^{P}(t) \mathbf{B}_{0,3,0}^{P}(\mathbf{r}),
\end{gathered}
$$

where $\mathbf{v}_{0}(\mathbf{r})$ is Poincare mode represented as an expansion in a hilberoth subspace such that $\mathbf{v}_{0}(\mathbf{r}) \mid=1$, $u(t)$ - the velocity amplitude, $\mathbf{B}_{0,1,0}^{P}(\mathbf{r})$ - the dipole component of the magnetic field, which generates a toroidal $\mathbf{B}_{0,2,0}^{T}(\mathbf{r})$ and a poloidal $\mathbf{B}_{0,3,0}^{P}(\mathbf{r})$ components under the influence of differential rotation [3]. The velocity field and magnetic field components are considered independent.

The function $Z(t)(5)$ is set as

$$
Z(t)=\int_{0}^{t}(t-\tau)^{n} e^{-b(t-\tau)} \mathbf{B}^{2}(\tau) d \tau,
$$


where the nature of the impact is determined by the exponential-power kernel $J(t)=t^{n} e^{-b t}$. In comparison with the exponential function of the form $e^{-b t}$, the maximum of the exponential-power function is shifted by the value $t_{0}$ - the delay time (Fig. 1), i. e., the influence of the process that inhibition the intensity of the $\alpha$-effect is turned on at the time shifted by the final delay time. The final waiting time $t_{m}=t_{1}-t_{0}$ is determined from the ratio

$$
\int_{t_{0}}^{t_{1}} t^{n} e^{-b t} d t=0.95 \int_{t_{0}}^{\infty} t^{n} e^{-b t} d t .
$$

Geometrically, this means that $95 \%$ of the area under the graph of the function is concentrated on this segment (Fig. 1).

Substituting in the system (6) decompositions (7), (8) and the function (9). After applying the Galerkin method, we get the system

$$
\begin{aligned}
& \frac{\partial u}{\partial t}=-P_{m} u(t) \sum_{k} \alpha_{k}^{2} \lambda_{k}+f_{\text {out }}+\sum_{i, j, k} \alpha_{i} L_{i j k} B_{j} B_{k}, \\
& \frac{\partial B_{i}}{\partial t}=R e_{m} u(t) \sum_{j, k} \alpha_{j} W_{i j k} B_{k}-\mu_{i} B_{i}+\left(R_{\alpha}-Z_{n}\right) \sum_{k} W_{i k}^{\alpha} B_{k}, \\
& \frac{\partial Z_{0}}{\partial t}=\sum_{k} B_{k}^{2}-b Z_{0}, \\
& \frac{\partial Z_{n}}{\partial t}=n Z_{n-1}-b Z_{n}, n=1,2, \ldots
\end{aligned}
$$

where $\mu_{i}$ is the coefficient of viscous dissipation, $\lambda_{i}$ is the eigenvalues of the Poincare mode, and the coefficients $L_{i j k}, W_{i j k}, W_{i j}^{\alpha}$ are the volume integrals of the fields under consideration. The implicit Euler and the explicit four-step Runge-Kutta methods are used for numerical implementation of the system (11). The sampling step $h$ is set to $10^{-5}$. The initial conditions for the system to exit the rest point at time $t=0$ and generate the remaining components of the magnetic field are accepted

$$
u(0)=1, B_{2}^{T}(0)=0, B_{1}^{P}(0)=1, B_{3}^{P}(0)=0, Z_{0}(0)=0, Z_{n}(0)=0, n=1,2, \ldots
$$

The control parameters of the model, the magnetic Reynolds number $R e_{m}$ and the amplitude of $\alpha$-effect $R_{\alpha}$, were set in the ranges $(0,1000]$ and $(0,100]$, respectively. The calculations were carried out for the values of the scale factor $b=0.1,0.5,1,5,10$ and the values of the exponent $n=1,2$. Both methods for given parameters of the model gave identical results, which are presented on the phase plane of the parameters $R e_{m}, R_{\alpha}$ (Fig. 2 b-f, Fig. 3).

The results obtained for $n=1$ show, that for the values of the scale coefficient $b$ of the kernel $J(t)$ of the function $Z(t)$, that do not exceed one, on the phase plane of the parameters $R e_{m}, R_{\alpha}$ the area of divergence of the magnetic field increases, and at $0.5 \leq b \leq 1$, the dynamo-burst regions appear with small inclusions of the regions of the regular mode of magnetic field generation. For values $b>1$, the region of steady mode generation becomes the same as it was for the case of constant intensity $\alpha$-effect $\alpha_{0}$, and the regions of steady-state and steady modes and vasillation mode appear at $0<R e_{m}<100$ and $0<R_{\alpha}<25$.

Consequently, as values of the scale coefficient $b$ increase, regions with the mode of dynamo-burst are gradually replaced by the regions of the steady mode (Fig. 2 c-f) and increase to the size of the steady mode generation region obtained with constant intesivity $\alpha_{0}$ (Fig. 2 a), and small regions of the steady-state mode and vasillation mode appear for $R_{m}<100, R_{\alpha}<25$. Such changes are related to the temporal characteristics of the function $Z(t)$ kernel. When the scale factor values are close to zero $(b<0.5)$, the values of the delay time $t_{0}$ and the waiting time (process memory) $t_{m}$ increase to the 
Table 1. Temporal characteristics of the function $J(t)=t^{n} e^{-b t}$

\begin{tabular}{|c|c|c|c|}
\hline$n$ & $b$ & delay time $t_{0}$ & waiting time $t_{m}$ \\
\hline \multirow{4}{*}{1} & 0.1 & 10 & 37.695207 \\
\cline { 2 - 4 } & 0.5 & 2 & 7.539041 \\
\cline { 2 - 4 } & 1 & 1 & 3.769521 \\
\cline { 2 - 4 } & 5 & 0.2 & 0.753904 \\
\cline { 2 - 4 } & 10 & 0.1 & 0.376952 \\
\hline \multirow{4}{*}{2} & 0.1 & 20 & 44.675701 \\
\cline { 2 - 4 } & 0.5 & 4 & 8.935140 \\
\cline { 2 - 4 } & 1 & 2 & 4.467570 \\
\cline { 2 - 4 } & 5 & 0.4 & 0.893514 \\
\cline { 2 - 4 } & 10 & 0.2 & 0.446757 \\
\hline
\end{tabular}

order of 10 and more (Table 1), and the value of the integral from $J(t)$ also increases. The impact of the process $Z(t)$ on the intensity of $\alpha$-effect with a long time delay $t_{0}$ leads to an unlimited increase in the magnetic field, i. e., the process does not have time to slow down the growth of the magnetic field. When $b \geq 1$, the values of the delay times $t_{0}$ and the waiting times $t_{m}$ quickly decrease from units to close to zero (Table 1). Such a small time shift and at the same time a small impact in comparison with the case of $b<1$ allows a turbulent effect with variable intensivity on the interval $R_{\alpha} \in(0,30]$ to bring the magnetic field to stable oscillations without unlimited increase, including with reversals. However, for the values of $R_{\alpha} \leq 10$ for all values of $b$, the turbulent generator does not output the magnetic field from the damped mode.

Consequently, under otherwise identical conditions of a numerical experiment, the variety of magnetic field generation modes at a weakly varying velocity field increases with an increase in the scale factor $b$, when the time of the gradual increasing impact of the process $Z(t)$ and force of that impact are decrease.

For exponent $n=2$, a pattern similar to the case of exponent $n=1$ is observed. Note, that here we obtain a damped mode and a mode of unlimited growth of the magnetic field already at $b<1$, when the increasing effect of the process $Z(t)$ is the largest and significantly prolonged over time. When the scale factor values $b>1$, the delay time $t_{0}<1$ and the waiting time are also reduced, which leads to small changes in the turbulent effect and the picture on the phase plane becomes similar to the case with a constant $\alpha$-effect intensity, only in the region where $\operatorname{Re}_{m}<100$, steady, steady-state and vacillation mode appear, which occupy smaller areas on the phase plane in comparison with the case when $n=1$.

Thus, the inclusion of an additive correction of the $\alpha$-effect intensity with the kernel as an exponential-power function leads to an increase in the region of the magnetic field divergence at a fixed exponent $n$ and at a decrease in the scale coefficient $b$, i. e., with an increase in the delay time. When the value $b$ increases, the delay time $t_{0}$ and the waiting time decrease, so the effect of the $Z(t)$ process on the $\alpha$-effect intensity becomes insignificant over time, and therefore the picture on the phase plane becomes similar to the case with constant intesivity $\alpha_{0}$. When the exponent $n$ increases and the respectively scale coefficients $b$ is equal, the delay time and the degree of the process impact on the $\alpha$-effect intensity increases, which leads to the predominance of two generation modes damped and unlimited growth, even when value of the coefficient $b$ increases. Besides, at the values of the parameter $R_{\alpha} \leq 10$, the additional inhibitory impact of the process $Z(t)$ does not lead to a decrease in the region of magnetic field attenuation at any values $n$ and $b$. 


\section{Conclusions}

In the framework of the accepted low-mode model $\alpha \Omega$-dynamo, a dimensionless MHD-system with an additive correction of the $\alpha$-effect intensity is considered under the assumption of axial symmetry of the velocity field and the magnetic field. The time scale is chosen equal to the time of magnetic field dissipation under the influence of an external force and in the absence of further additional influences. The influence of the turbulent $\alpha$-effect is determined by a function $Z(t)$ with an exponential-power kernel of the form $t^{n} e^{-b t}$, which specifies a process with a delay time of $t_{0}$ and a limited waiting time of $t_{m}$.

The results of calculations showed that the inclusion of the process $Z(t)$ into the MHD-system leads to the appearance of new modes of magnetic field generation in comparison with the case of constant intensity $\alpha_{0}$, including with reversals. When the delay time $t_{0}$ decreases (the scale factor $b$ increases), the region of non-divergent oscillations increases. An increase in the exponent $n$ is associated with an increase in the delay time $t_{0}$ and leads to an expansion of the region of unlimited oscillations at an increase in the scale coefficient $b$.

Thus, an increase in the values of the temporal characteristics of the exponential-power kernel $J(t)$ reduces the number of generation modes with a predominance of an unlimited increase the magnetic field, but practically does not affect the region of damped oscillations, only slightly increases it at values $b<1$. Reducing the values of the temporal characteristics of the kernel $J(t)$ increases the number of generated magnetic field modes and reduces the region of unlimited increase in the magnetic field, and the steady oscillations at $R e_{m}>100$ gradually occupy the same region on the phase plane as at constant intensity, gradually slightly increasing it in the direction of increasing the values of $R_{\alpha}$.

The paper was carried out within the framework on the subject «Dynamics of physical processes in active zones of near space and geospheres» (AAAA-A17-117080110043-4)

\section{References}

[1] M. Steenbek, F. Krause, Astron. Nachr. 291, 49-84 (1969)

[2] Ya.B. Zeldovich, A.A. Rusmaikin, D.D. Sokoloff, Magnetic fields in astrophysics. The Fluid Mechanics of Astrophysics and Geophysics (Gordon and Breach, New York, 1983) 382

[3] G.M. Vodinchar, L.K. Feshchenko, Bulletin KRASEC. Phys. and Math. Sci, Mathematical modeling, 2(11), 42-54 (2015)

[4] G.M. Vodinchar, Bulletin KRASEC. Phys. and Math. Sci, Mathematical modeling, 2(7), 33-42 (2013)

[5] E.N. Parker, Astrophys. J., 122, 293-314 (1955)

[6] A.N. Godomskaya, O.V. Sheremetyeva, E3S Web of Conferences, 62, 02016 (2018)

[7] G.M. Vodinchar, A.N. Godomskaya, O.V. Sheremetyeva, Bulletin KRASEC. Phys. and Math. Sci, Mathematical modeling, 2(7), 33-42 (2018)

[8] A.N. Godomskaya, O.V. Sheremetyeva, E3S Web of Conferences, 127, 02016 (2019)

[9] A.N. Godomskaya, O.V. Sheremetyeva, Bulletin KRASEC. Phys. and Math. Sci, Mathematical modeling, 4(29), 58-66 (2019) 


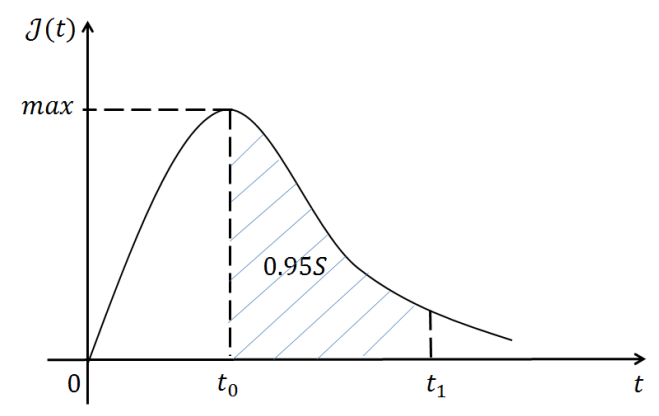

Figure 1. The exponential-power function $J(t)$ graph. 


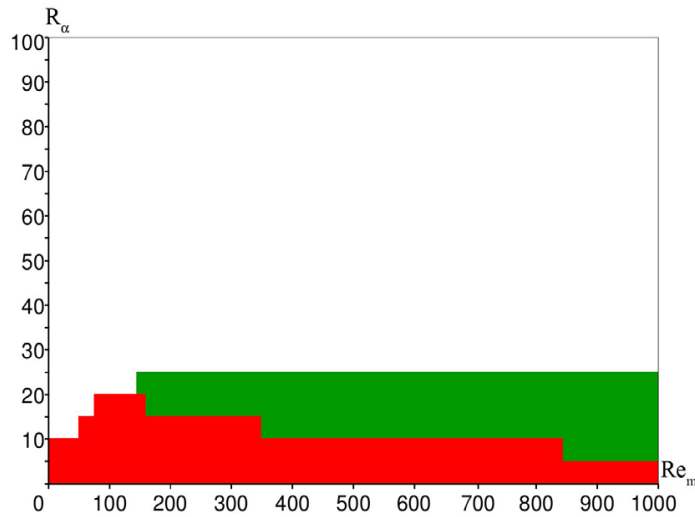

a)

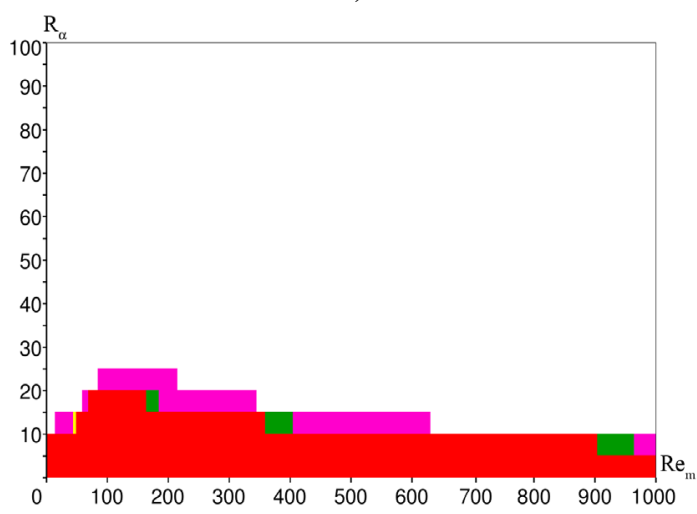

c)

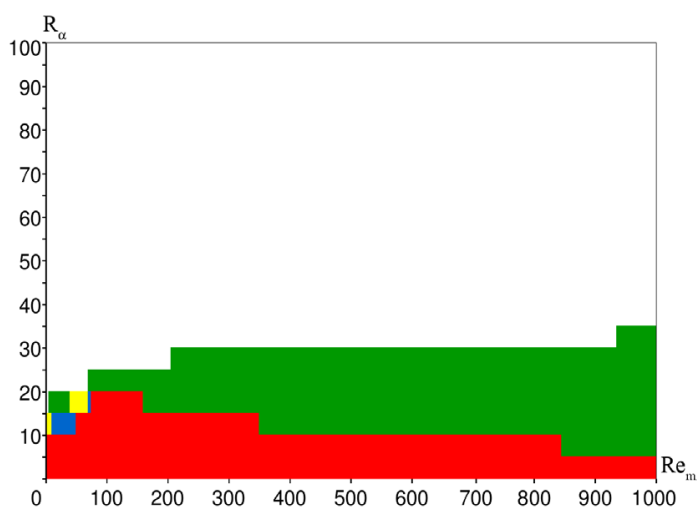

e)

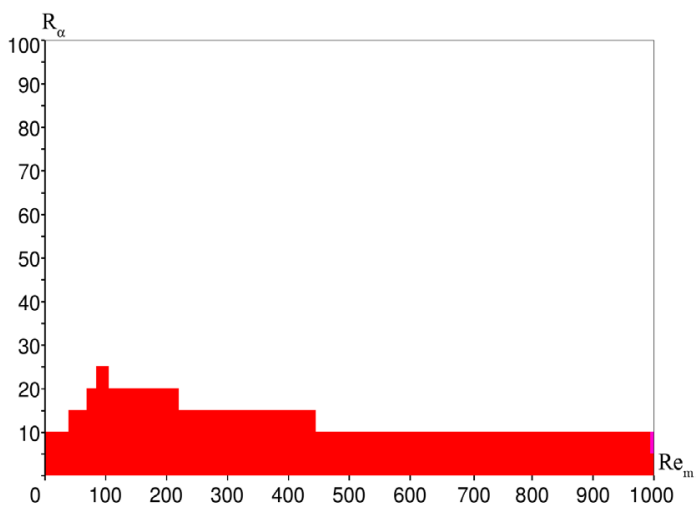

b)

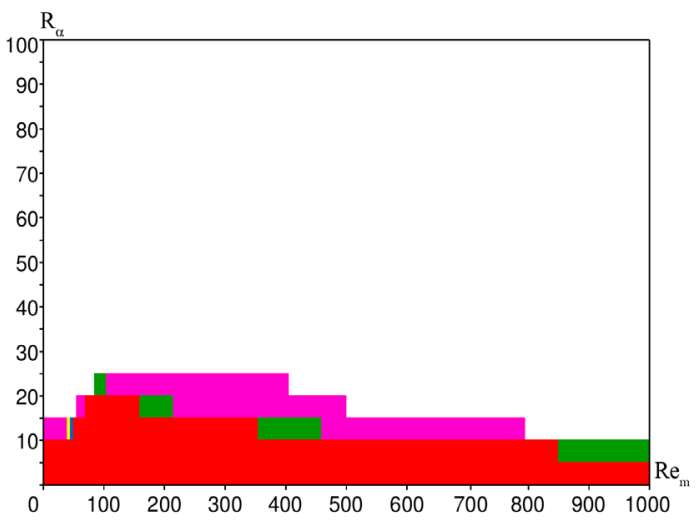

d)

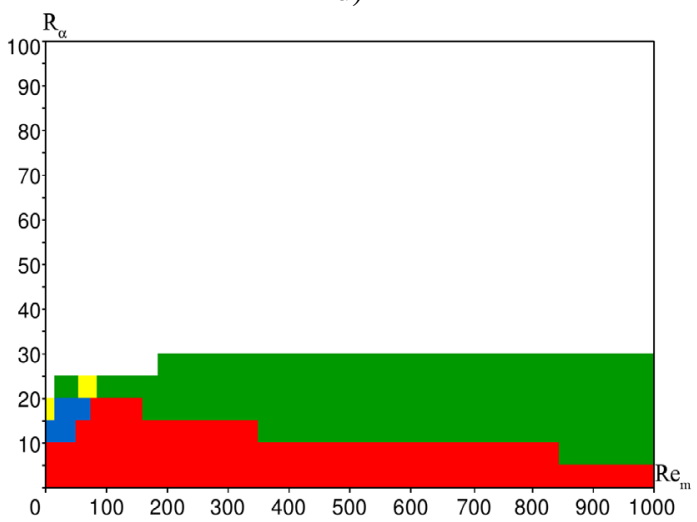

f)

Figure 2. The nature of magnetic field generation depending on the parameters $R_{\alpha}$ and $R e_{m}$. The $\alpha$-effect intensity: a) constant $-\alpha_{0}$; in other cases is defined by the function $Z(t)$ with the kernel: b) $J(t)=t e^{-0,1 t}$, c) $J(t)=t e^{-0,5 t}$, d) $J(t)=t e^{-t}$, e) $J(t)=t e^{-5 t}$, f) $J(t)=t e^{-10 t}$. The white region is the generation of the increases infinitely magnetic field, the red one - is the generation of a damped magnetic field, the green is the steady mode of magnetic field generation, the blue is the steady-state mode, the yellow is the vacillation, the lilac is the dynamo-burst. 


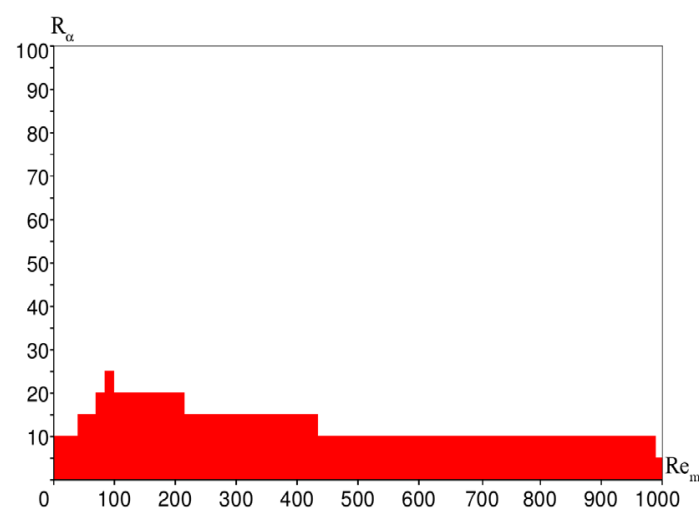

a)

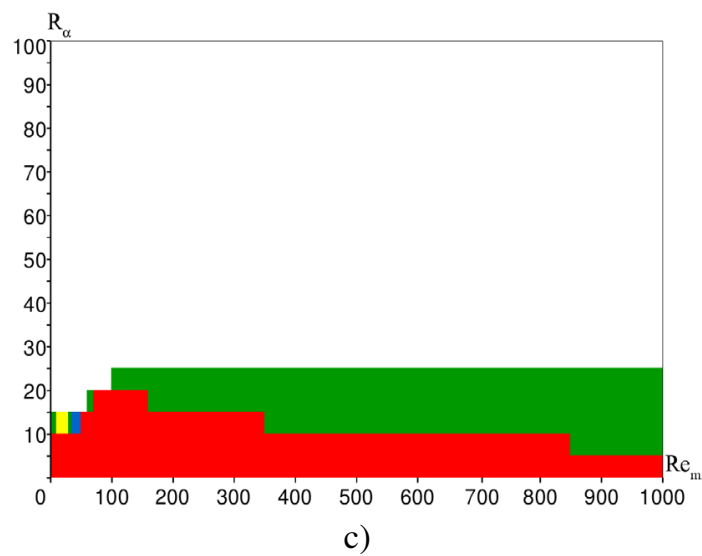

Figure 3. The nature of magnetic field generation depending on the parameters $R_{\alpha}$ and $R e_{m}$. The $\alpha$-effect intensity is defined by the function $Z(t)$ with the kernel: a) $J(t)=t^{2} e^{-0,5 t}$, b) $J(t)=t^{2} e^{-t}$, c) $J(t)=t^{2} e^{-5 t}$, d) $J(t)=t^{2} e^{-10 t}$. The white region is the generation of the increases infinitely magnetic field, the red one - is the generation of a damped magnetic field, the green is the steady mode, the blue is the steady-state mode, the yellow is the vacillation, the lilac is the dynamo-burst. 